\title{
Prevalência de características da oclusão normal na dentição decídua
}

\section{Prevalence of normal occlusal traits in deciduous dentition}

\author{
Rivea Inês FERREIRA* \\ Alice Kelly BARREIRA** \\ Carolina Dias SOARES** \\ Alessandra Castro ALVES***
}

\begin{abstract}
FERREIRA, R. I.; BARREIRA, A. K.; SOARES, C. D.; ALVES, A. C. Prevalência de características da oclusão normal na dentição decídua. Pesqui Odontol Bras, v. 15, n. 1, p. 23-28, jan./mar. 2001.

O objetivo deste estudo foi verificar os padrões oclusais normais da dentição decídua em crianças pré-escolares brasileiras. A amostra foi constituída por 356 crianças, de ambos os sexos, na faixa etária de 3-5 1/2 anos e residentes na cidade de Salvador. O exame de oclusão foi realizado na sala de aula, por duas examinadoras, utilizando-se uma espátula de madeira. Os dados foram analisados pelo EPI-INFO 6.02, aplicando-se o teste $\chi^{2}$. A freqüência do arco tipo I foi de $43,3 \%$ para a arcada superior e $46,3 \%$ para a inferior. O arco tipo II esteve presente em $56,7 \%$ das arcadas superiores e em $53,7 \%$ das inferiores, entretanto, sem diferença entre os sexos. A distribuição dos espaços primatas foi de $89,9 \%$ para o arco superior e $67,1 \%$ no arco inferior, diminuindo significativamente tal prevalência com o aumento das idades $(\mathrm{p}<0,01)$. A relação canina normal foi encontrada em quase $60 \%$ das crianças, para ambos os lados. Dos 712 planos terminais distais avaliados, 55,9\% apresentaram plano terminal mesial para os segundos molares decíduos; $37,9 \%$ plano terminal reto e $6,2 \%$ degrau distal. Os resultados permitem concluir que a presença dos espaços interincisais generalizados, comumente relatados por outros autores, não foi o mais freqüente para ambos os arcos nesta amostra, contudo, os espaços primatas foram os mais prevalentes. Os freqüentes padrões normais de oclusão para as relações canino e molar foram os de Classe I.
\end{abstract}

UNITERMOS: Dentição primária; Oclusão dentária; Ortodontia preventiva.

\section{INTRODUÇÃO}

Muito embora não se tenha estabelecido seguramente o efeito que os padrões de oclusão da dentição decídua podem determinar sobre o desenvolvimento da oclusão satisfatória dos dentes permanentes ${ }^{12}$, vários são os trabalhos que procuram analisar as características da dentição decídua e correlacioná-las a possiveis expressões na dentição permanente ${ }^{4,10,12,14,15,16,17,19,21,23}$.

Os espaços interdentários localizados na secção anterior do arco decíduo foram primeiramente descritos por Delabarre, em 1819 ${ }^{2,9,12}$. Este pesquisador sugeriu que a finalidade desse espaçamento era propiciar uma compensação para o diâmetro mésio-distal dos dentes permanentes. BAUME ${ }^{2}$, em 1950, observou dois tipos de arcos decíduos: arco tipo I, apresentando espaços generalizados entre os dentes na região anterior, e arco tipo II, sem espaços, os quais seriam mais estreitos transversalmente. Esta característica pode ser comprovada pela determinação das distâncias intercanino e intermolar, como foi demonstrado por GAIBOR ${ }^{5}$, em 1986. Quanto à freqüência, $\mathrm{BAUME}^{2}$ verificou que $70 \%$ das crianças eram portadoras de arcos tipo I e 30\% do tipo II na maxila. Para a mandíbula, a freqüência era de $63 \%$ de crianças com arcos do tipo I e 37\% do tipo II.

Nesse mesmo estudo, BAUME ${ }^{2}$ (1950) constatou a presença de diastemas característicos, no arco superior, entre incisivos laterais e caninos decíduos; no arco inferior, entre caninos e primeiros molares decíduos. Esses diastemas foram denominados de espaços primatas. Em ambos os arcos, e particularmente nos do tipo II, a presença dos es-

\footnotetext{
* Acadêmica do curso de Odontologia e Bolsista de Iniciação Científica do PIBIC; ** Estagiárias da Disciplina de Odontopediatria - Faculdade de Odontologia da UFBA.

*** Professora Assistente da Disciplina de Odontopediatria da Faculdade de Odontologia da UFBA e Faculdade de Odontologia da UEFS.
} 
FERREIRA, R. I.; BARREIRA, A. K.; SOARES, C. D.; ALVES, A. C. Prevalência de características da oclusão normal na dentição decídua. Pesqui Odontol Bras, v. 15, n. 1, p. 23-28, jan./mar. 2001.

paços primatas é altamente favorável para o bom alinhamento dos dentes permanentes anteriores ${ }^{7,9}$, 12, 15,18. Entretanto, os espaçamentos são muito variáveis, podendo haver uma ausência total ou uma presença generalizada ${ }^{9,15}$.

Em 1987, USBERTI; CUNHA ${ }^{18}$, em um outro trabalho que tinha por objetivo verificar a prevalência de indivíduos portadores dos arcos tipos I e II, assim como a presença de espaços primatas, em uma amostra de crianças portadoras de relação terminal em plano reto e degrau mesial, concluíram que o arco tipo I é mais freqüente na arcada superior, tanto nos indivíduos portadores de relação terminal em plano vertical como em degrau mesial para a mandíbula; ao passo que o arco tipo II é mais freqüente na mandibula. Os tipos de arcos e os espaços primatas são características independentes na dentadura decídua, podendo estar presentes ou não, associados ou isolados na arcada dentária superior ou inferior ${ }^{18}$.

A dentição decídua estaria completa depois que os segundos molares entrassem em oclusão, geralmente por volta dos 2,5 anos de idade . BAUME $^{2}$ (1950), analisando os arcos decíduos superiores e inferiores pelas faces distais dos segundos molares decíduos, verificou que este relacionamento mantinha-se constante na faixa etária de 3 a 5 anos, da mesma forma que a relação canina, podendo ser classificado como plano terminal reto, degrau mesial ou degrau distal. A freqüência da relação terminal em plano reto era de $76 \%$; os degraus mesial e distal para a mandibula eram observados, respectivamente, em $14 \%$ e $10 \%$ dos casos.

Segundo ARYA et al. ${ }^{1}$ (1973), a oclusão inicial dos primeiros molares permanentes não seria completamente influenciada pela relação de plano terminal dos segundos molares decíduos. Contudo, reconhece-se que a relação das faces distais dos segundos molares decíduos serve como guia para a oclusão dos primeiros molares permanentes $^{2,3,8,10,11,20,21}$.

BURDI; MOYERS ${ }^{3}$ (1991), em seus estudos, demonstraram a preponderância do padrão de crescimento esquelético sobre os mecanismos de ajuste da dentição. Um degrau distal na dentição decídua refletiria um provável desequilíbrio esquelético, que certamente resultará em uma maloclusão de Classe II na dentição permanente. De acordo com este pesquisador, os dois caminhos mais comuns, e portanto normais, seriam de um plano terminal reto para a relação topo a topo, e posteriormente relação em chave de oclusão ou de de- grau mesial para o engrenamento dos primeiros molares permanentes.

As pesquisas de BAUME ${ }^{2}$ (1950) mostraram um relacionamento oclusal de segundos molares decíduos inalterado durante o período da dentição decídua. No entanto, em determinados grupos étnicos que consomem uma dieta fibrosa, um degrau mesial poderia formar-se a partir de um plano terminal reto, devido a uma maior atrição e remoção de interferências cuspídeas ${ }^{3,11}$, o que permitiria à mandíbula assumir facilmente uma posição mais anterior na faixa etária de 5 a 6 anos, época em que este osso cresce mais que a maxila ${ }^{3}$.

Os trabalhos de VALENTE et al. ${ }^{20}$ (1978) demonstraram que em crianças nas faixas etárias extremas, isto é, de 2 a 3 anos e de 5 a 6 anos, o plano terminal reto e o degrau mesial ocorrem com maior freqüência do que a relação terminal em degrau distal. Os referidos autores também observaram que a relação terminal de segundos molares decíduos não apresentou diferença significativa entre crianças do sexo masculino e feminino.

WOON $^{22}$ (1988), pesquisando a prevalência das características da dentição decídua em chineses, malasianos e indianos, verificou que os indianos de sua amostra não possuíam relação de segundos molares decíduos em degrau mesial. Entretanto, as percentagens de individuos com plano terminal reto e degrau distal eram de $62 \%$ e $38 \%$, respectivamente. O degrau mesial podia ser observado, ainda que em menores percentagens que as outras relações terminais, nos chineses e malasianos.

Outros estudos no Brasil estudaram a relação distal dos segundos molares decíduos em crianças. Constataram que o plano terminal reto podia ser encontrado em $64,8 \%$ da amostra. O degrau mesial estava presente em $34,6 \%$ das crianças e o degrau distal em $0,6 \%{ }^{14}$.

MOURA et al. ${ }^{10}$ (1994) verificaram uma maior prevalência da relação terminal em plano reto dos segundos molares decíduos, em $81,9 \%$ dos lados avaliados, seguida por degrau mesial e degrau distal, que corresponderam a $13,2 \%$ e $4,9 \%$, respectivamente.

Um estudo realizado no sul da Índia, em 1998, com 1.026 crianças entre 3 e 4 anos de idade teve como proposição avaliar o perfil, as relações oclusais e a presença ou não de espaçamento anterior. A relação plana dos segundos molares decíduos foi a mais encontrada para ambos os sexos. A mesma associou-se significativamente com o perfil convexo, o mesmo ocorrendo com a relação distal, quando a mesma esteve presente. Setenta e cinco por- 
FERREIRA, R. I.; BARREIRA, A. K.; SOARES, C. D.; ALVES, A. C. Prevalência de características da oclusão normal na dentição decídua. Pesqui Odontol Bras, v. 15, n. 1, p. 23-28, jan./mar. 2001.

cento das crianças de ambos sexos apresentaram espaçamento fisiológico e espaços primatas em ambos os $\operatorname{arcos}^{15}$.

\section{PROPOSIÇÃO}

A partir do exame clínico e seleção de crianças pré-escolares, com oclusão satisfatória, esta pesquisa teve como proposição identificar a prevalência das características da dentição decídua que influenciam preponderantemente a oclusão permanente, tais como: tipo de arco, presença de espaços primatas, relação terminal de segundos molares e relação de caninos decíduos.

\section{CASUÍSTICA E MÉTODO}

Para a realização deste estudo, foram examinadas 693 crianças na faixa etária de 3 a 5 1/2 anos de idade $(4,05 \pm 0,77)$, saudáveis e provenientes de 14 escolas particulares localizadas nos principais bairros de classe média da cidade de Salvador (BA). A amostra estudada constou de 356 crianças portadoras de dentição decídua completa, selecionadas com base nos seguintes critérios de exclusão $0^{13,15,16}$ :

- presença de mordidas abertas, sobremordida exagerada ou mordidas cruzadas;

- lesões de cárie ou restaurações proximais que comprometessem os diâmetros mésio-distais dos dentes;

- perdas precoces de dentes ou dentição decídua incompleta;

- anomalias de forma, número, estrutura e irrupção.

As informações referentes ao nome, gênero e data de nascimento foram coletadas das fichas de matrícula escolar de cada criança. Posteriormente, procedeu-se ao exame clínico. Este procedimento foi realizado por duas examinadoras, cirurgiãsdentistas, estagiárias da disciplina de Odontopediatria da Universidade Federal da Bahia, previamente calibradas, auxiliadas por uma assistente. Para tanto, utilizaram-se carteiras escolares comuns, luz ambiente, espátulas de madeira descartáveis e fichas clínicas especialmente elaboradas para esta pesquisa.

A inspeção dos arcos ocorreu em duas fases. Inicialmente, a criança era examinada em posição de relaxamento, possibilitando a classificação dos arcos em tipo I ou tipo II, segundo BAUME ${ }^{2}$ (1950). As examinadoras, utilizando-se de um fio ortodôntico redondo de $0,5 \mathrm{~mm}$ de espessura como elemento de comparação, consideravam os espaços menores que esta medida ausentes e, arcos com pelo menos um dos diastemas desconsiderado eram classificados como tipo $\mathrm{II}^{3,16}$.

$\mathrm{Na}$ segunda fase, a criança permanecia em posição de máxima intercuspidação habitual, para que se pudesse observar as relações de molares e caninos decíduos. As características foram analisadas no programa EPI-INFO 6.02. O teste do qui-quadrado foi aplicado para viabilizar a comparação das relações normais de oclusão na amostra estudada, com um nivel de significância de 1\%.

\section{RESULTADOS E DISCUSSÃO}

Para avaliar as características que refletissem a situação de desenvolvimento ideal em uma amostra homogênea, foram selecionadas 356 crianças de ambos os gêneros, $52 \%$ meninas e $48 \%$ meninos, de um total de 693 examinadas. As crianças eram estudantes de 14 escolas particulares da cidade de Salvador e estavam na faixa etária de 3 a 5 $1 / 2$ anos, sendo que $27,5 \%, 39,3 \%$ e $33,1 \%$ tinham 3, 4 e 5 anos, respectivamente. A opção por fazer o levantamento em instituições privadas deu-se em função da proposta fundamental do trabalho, que era estudar a prevalência de características da oclusão normal sob condições presumivelmente apropriadas de desenvolvimento, uma vez que as crianças estavam condicionadas à influência de padrões socioeconômicos, nutricionais e educacionais semelhantes.

O Gráfico 1 mostra que, ao contrário da literatura revisada ${ }^{2,15,18}$, os arcos tipo II foram os mais prevalentes em ambas as arcadas. USBERTI; CUNHA $^{18}(1987)$ também observaram a maior prevalência do arco tipo II, porém, apenas na mandíbula. A alta prevalência do arco tipo II, neste estudo, pode ter sido decorrente da metodologia aplicada. Tendo em vista que a discrepância entre o tamanho das bases ósseas apicais e os diâmetros

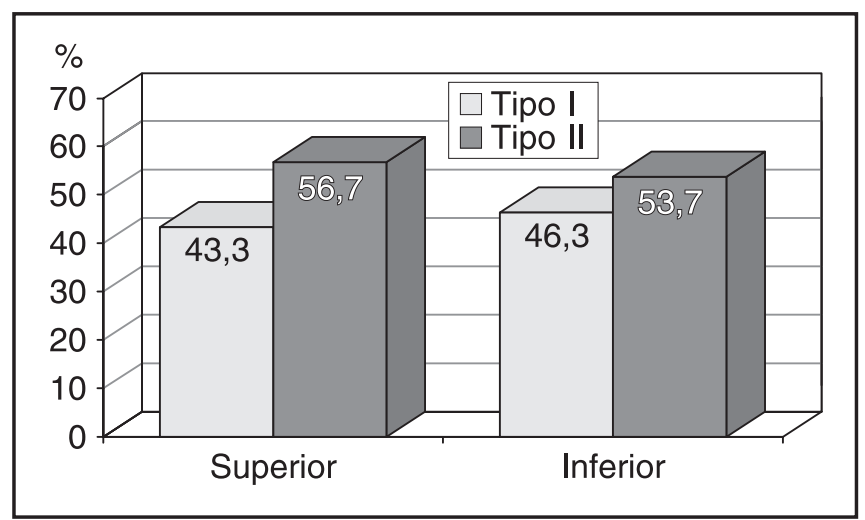

GRÁFICO 1 - Prevalência de arcos tipo I e II de Baume nos arcos superiores e inferiores. 
FERREIRA, R. I.; BARREIRA, A. K.; SOARES, C. D.; ALVES, A. C. Prevalência de características da oclusão normal na dentição decídua. Pesqui Odontol Bras, v. 15, n. 1, p. 23-28, jan./mar. 2001.

mésio-distais das respectivas unidades dentárias pode ocasionar apinhamentos, giroversões e irrupções ectópicas ${ }^{17,21,23}$, procurou-se avaliar não só a presença de espaços generalizados, mas também a largura dos mesmos.

Quanto à distribuição dos espaços primatas, como pode-se observar a partir da análise dos Gráficos 2 e 3, estes diastemas eram mais freqüentes nos arcos superiores que nos inferiores, sendo menos prevalentes nas crianças com idade mais avançada $(p<0,01)$. A tendência para o fechamento dos espaços primatas pode ser explicada pela irrupção dos dentes permanentes, que em geral ocorre inicialmente na arcada inferior, e pela ação da componente vetorial anterior da força de mastigação que se estabelece com o desenvolvimento da oclusão $0^{3,6,16,17,23}$.

A interpretação do Gráfico 4 permite inferir que, a despeito das assimetrias entre os lados direito e esquerdo, a relação terminal dos segundos molares decíduos em degrau mesial foi predominante, seguida pelas relações em plano vertical ou reto e degrau distal. Em se tratando de uma amostra

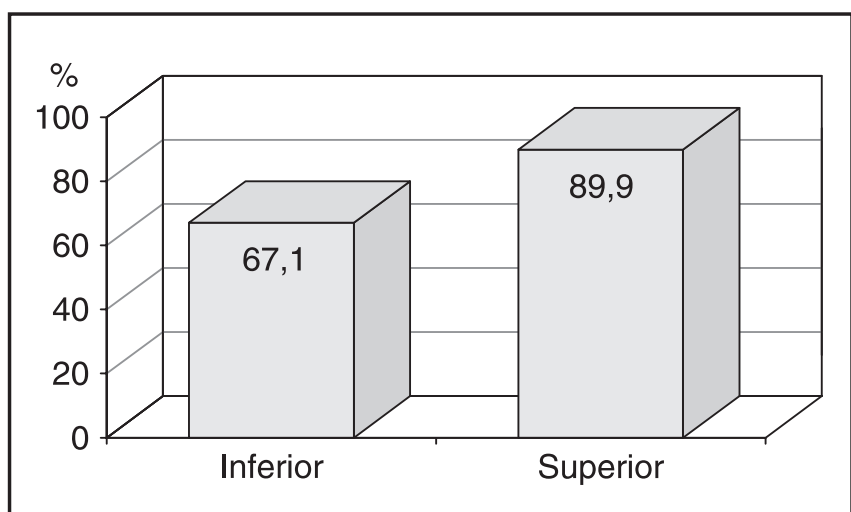

GRÁFICO 2 - Distribuição dos espaços primatas nos arcos superiores e inferiores.

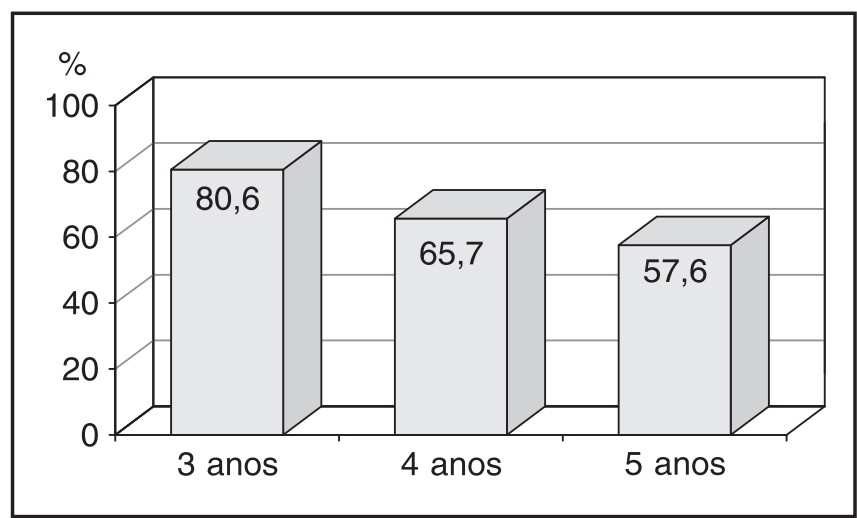

GRÁFICO 3 - Freqüência dos diastemas primatas inferiores, de acordo com a idade. constituída majoritariamente por crianças de 4 a $51 / 2$ anos, é natural que possa ter ocorrido uma alteração nas relações devida a fatores como o crescimento mandibular ${ }^{3}$, a eliminação de interferências cuspídeas pela mastigação de alimentos mais fibrosos ${ }^{3,11,17}$ e o início dos movimentos irruptivos dos primeiros molares permanentes. Estes acontecimentos evolutivos poderiam ter contribuído para a substituição do plano terminal reto pelo degrau mesial.

Outro dado importante e, no entanto, pouco pesquisado ${ }^{15}$ é a relação de oclusão dos caninos decíduos. Como ilustra o Gráfico 5, a relação normal esteve presente em mais da metade dos lados examinados, seguida pelas relações em topo e distal, que foi pouco significativa.

Ao comparar os resultados deste estudo com o achados de outros autores ${ }^{6,15,19,20,22}$, pode-se depreender que os fatores ambientais, nutricionais, socioeconômicos e educacionais podem influenciar substancialmente o desenvolvimento da oclusão na dentição decídua, não sendo o sexo um elemento de significância.

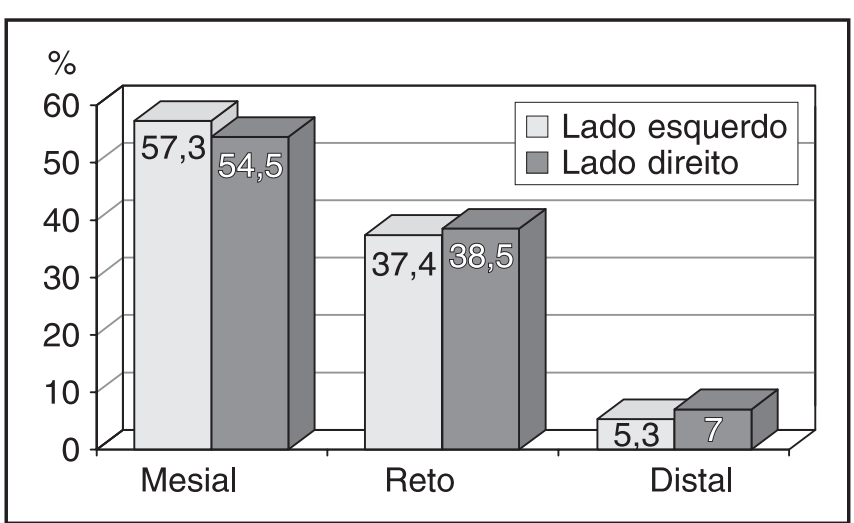

GRÁFICO 4 - Freqüência dos tipos de relações terminais dos segundos molares decíduos, para ambos os lados.

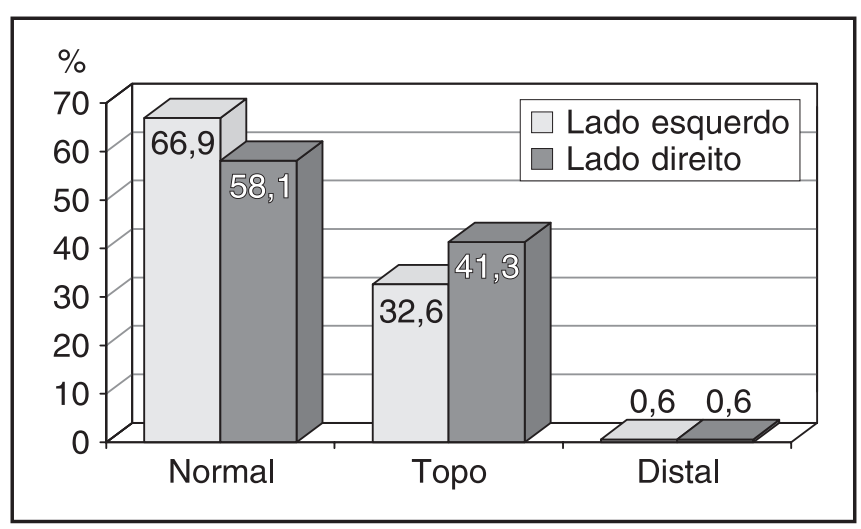

GRÁFICO 5 - Distribuição dos pré-escolares segundo a relação de caninos. 
FERREIRA, R. I.; BARREIRA, A. K.; SOARES, C. D.; ALVES, A. C. Prevalência de características da oclusão normal na dentição decídua. Pesqui Odontol Bras, v. 15, n. 1, p. 23-28, jan./mar. 2001.

\section{CONCLUSÕES}

1. O tipo de arco mais prevalente foi o II, tanto para a arcada superior quanto para a inferior, em ambos os sexos.
2. Os espaços denominados primatas foram mais prevalentes nas crianças menores de 4 anos.

3. Os padrões normais de oclusão mais freqüentes, para as relações canino e molar, foram os de Classe I.

FERREIRA, R. I.; BARREIRA, A. K.; SOARES, C. D.; ALVES, A. C. Prevalence of normal occlusal traits in deciduous dentition. Pesqui Odontol Bras, v. 15, n. 1, p. 23-28, jan./mar. 2001.

The aim of this research was to study the normal occlusal patterns of deciduous dentition in Brazilian preschool children. The sample consisted of 356 preschool children, of both genders, aged 3-5 1/2 years from the urban zone of Salvador, Bahia. The occlusion exam was carried out by two examiners, in the classroom, utilizing a wooden spatula. The data were analyzed by the statistical program EPI-INFO 6.02 using the Chi-squared test. The frequency of the type I arch was $43.3 \%$ for the upper and $46.3 \%$ for the lower arch. The frequency of the type II arch was $56.7 \%$ for the upper and 53.75 for the lower arch, however, no difference between genders was noticed. The distribution of primate spaces was: $89.9 \%$ for the upper and $67.1 \%$ for the lower arch. This prevalence decreased significantly as age increased $(\mathrm{p}<0.01)$. Normal canine relationship was found in almost $60 \%$ of the children for the right and left sides. Out of 712 evaluated terminal planes, $55.9 \%$ presented mesial terminal step for the primary second molars, $37.9 \%$ straight terminal plane and $6.2 \%$ distal step. The results permitted to conclude that the presence of generalized spacing between incisors, commonly described by other authors, was not the most prevailing condition for both arches in this sample. On the other hand, primate spaces were the most common ones. The most frequent normal occlusal patterns for canine and molar relationships were found to be the Class I patterns.

UNITERMS: Dentition, primary; Dental occlusion; Orthodontics, preventive.

\section{REFERÊNCIAS BIBLIOGRÁFICAS}

1. ARYA, B. S.; SAVARA, B. S.; THOMAS, D. R. Prediction of first molar occlusion. Am J Orthod, v. 63, n. 6, p. 610-621, June 1973.

2. BAUME, L. J. Physiological tooth migration and its significance for the development of occlusion. I - the biogenetic course of the deciduous dentition. J Dent Res, v. 29, n. 2, p. 123-132, Apr. 1950.

3. BURDI, A. R.; MOYERS, R. E. Desenvolvimento da dentição e da oclusão. In: MOYERS, R. E. Ortodontia. 4. ed., Rio de Janeiro: Guanabara Koogan, 1991. p. $86-126$.

4. CAMBEROS, C. E. R.; CHELOTTI, A.; ANDO, T. Forma do arco dental decíduo, tipo I e II de Baume e as possíveis diferenças quanto ao sexo e lado. Rev Odontol Univ São Paulo, v. 5, n. 2, p. 106-111, jul./dez. 1991.

5. GAIBOR, M. G. C. Estudo comparativo das distâncias intercanina e intermolar, em arcos tipo I e II de Baume, na faixa etária de $\mathbf{3}$ a $\mathbf{5}$ anos. São Paulo, 1986. 48 p. Dissertação (Mestrado em Odontopediatria) Faculdade de Odontologia, Universidade de São Paulo.

6. GANDINI, M. R. E. A. S.; PINTO, A. S.; GANDINI Jr., L. G.; MARTINS, J. C. R.; MENDES, A. J. D. Estudo da oclusão dentária de escolares da cidade de Araraquara, na fase da dentadura mista. Ortodontia, v. 27, n. 3, p. 37-48, set./dez. 1994.

7. GAVIÃO, M. B. D.; CHELOTTI, A. Estudo comparativo dos arcos dentários decíduos espaçados e não espaçados: segmento anterior. Rev Odontopediatr, v. 1, n. 3, p. 195-201, jul./set. 1992.

8. LINDEN, F. P. G. M. Ortodontia: desenvolvimento da dentição. São Paulo : Quintessence Books, 1986. 206 p.
9. LOOR, L. C. V. Contribuição para o estudo da angulagem existente entre as faces oclusais dos primeiros e segundos molares decíduos inferiores e superficies incisais dos dentes decíduos anteriores inferiores, com relação ao plano horizontal, em pacientes portadores de arcos do tipo I e II de Baume, em ambos os sexos, na faixa etária de 3 a 5 anos. São Paulo, 1989. 52 p. Dissertação (Mestrado em Odontopediatria) - Faculdade de Odontologia, Universidade de São Paulo.

10. MOURA, M. S.; SIMPLÍCIO, A. H. M.; MOURA, L. F. A. D.; MOURA, W. L. Alterações na relação molar entre as dentaduras decídua e mista. Rev ABO Nac, v. 2, n. 5, p. 333-339, out./nov. 1994.

11. NANDA, R. S.; KHAN, I.; ANAND, R. Age changes in the occlusal pattern of deciduous dentition. J Dent Res, v. 52, n. 2, p. 221-224, Mar./Apr. 1973.

12. PACE, R. S. G.; CHELOTTI, A. Freqüência de distribuição dos espaços primatas em crianças portadoras do arco tipo I e II. Rev Odontol Univ São Paulo, v. 19, n. 1, p. 53-62, jan./jun. 1981.

13. ROUleT, P. L. B. C.; CHELOTTI, A. Contribuição para o estudo espacial da curvatura ântero-posterior dos arcos dentários decíduos tipo I e II de Baume, superior e inferior, projetada no plano sagital (curva de Spee). Rev Odontol Univ São Paulo, v. 9, n. 1, p. 45-50, jan./mar. 1995.

14. SERRA-NEGRA, J. M. C.; SERRA-NEGRA, E. Relação distal dos segundos molares decíduos. Arq Centro Estud Curso Odontol, v. 30, n. 1/2, p. 9-15, jan./dez. 1993-1994.

15. SHERENE, A.; NEETA, T. P. Profiles, occlusal plane relationships and spacing of teeth in the dentitions of 3 to 
FERREIRA, R. I.; BARREIRA, A. K.; SOARES, C. D.; ALVES, A. C. Prevalência de características da oclusão normal na dentição decídua. Pesqui Odontol Bras, v. 15, n. 1, p. 23-28, jan./mar. 2001.

4-year-old children. J Clin Pediatr Dent, v. 22, n. 4, p. 329-334, 1998.

16. STRANG, R. H. W. A text-book of Orthodontia. 4. ed., Philadelphia : Lea \& Febiger, 1958. $867 \mathrm{p}$.

17. TSUJINO, K.; MACHIDA, Y. A longitudinal study of the growth and development of the dental arch width from childhood to adolescence in Japanese. Bull Tokyo Dent Coll, v. 39, n. 2, p. 75-89, 1998.

18. USBERTI, A. C.; CUNHA, J. C. M. Freqüência de arcos tipo I e II de Baume e espaços primatas. RGO, v. 35, n. 6, p. 474-478, nov./dez. 1987.

19. USBERTI, A. C.; PETERS, C. F.; ISSÁO, M. Freqüência de arcos tipo I e II, em crianças com dentição decídua clinicamente normal. Rev Odontol Univ São Paulo, v. 19, n. 1, p. 71-77, jan./jun. 1981.
20. VALENTE, A.; RIBEIRO, Z. M. M.; CORDEIRO, R. C. L. et al. Relação ântero-posterior dos arcos dentais na dentição decídua - relação molar. Rev Fac Farm Odontol Ribeirão Preto, v. 15, n. 2, p. 115-122, jul./dez. 1978.

21. VARRELA, J. Early developmental traits in class II malocclusion. Acta Odontol Scand, v. 56, n. 6, p. 375-377, 1998.

22. WOON, K. C. Primary dentition occlusion in Chinese, Indian and Malay groups in Malaysia. Aust Orthod J, v. 10, n. 3, p. 183-185, 1988.

23. YOSHIHARA, T.; MATSUMOTO, Y.; SUZUKI, J. et al. Effect of serial extraction alone on crowding: relationships between tooth width, arch length and crowding. Am J Orthod Dentofacial Orthop, v. 116, n. 6, p. 691-696, 1999.
Recebido para publicação em13/12/99 Enviado para reformulação em 27/04/00 Aceito para publicação em 27/11/00 